\title{
The Correlations between Cord Blood Leptin and Leptin Level at Six Months with Infant Growth
}

\author{
Ika Rara Rosita ${ }^{1}$, Agustini Utari², Maria Mexitalia ${ }^{3 *}$ \\ ${ }^{1}$ Department of Pediatrics, Faculty of Medicine, Universitas Diponegoro, Semarang, Indonesia \\ ${ }^{2}$ Division of Pediatric Endocrinology, Department of Pediatrics, Faculty of Medicine, Universitas Diponegoro, \\ Semarang, Indonesia \\ ${ }^{3}$ Division of Nutrition and Metabolism, Department of Pediatrics, Faculty of Medicine, Universitas Diponegoro/ \\ Dr. Kariadi General Hospital, Semarang, Indonesia
}

\section{Article Info}

History

Received: 25 July 2021

Accepted: 27 Aug 2021

Available: 31 Aug 2021

\begin{abstract}
Background: Leptin plays an important role in regulating body weight, metabolism, and reproductive functions. Leptin affects metabolism by reducing nutrient intake and increasing energy expenditure which eventually also plays a role in infant growth.

Objective: This study aims to determine the relationship between leptin levels and infant growth age 0-6 months.

Methods: A prospective single cohort study was done for six months on newborns from breastfeeding mothers with normal pregnancies. The samples were taken twice, firstly when the infant was born using an umbilical cord blood sample, and secondly at the age of six months, using a vein blood sample. Serum leptin levels were measured using the ELISA method. Infant growth was assessed using WHO 2005 's z-scores.

Results: A total of 50 infants were included in the study, 38 of them completed all measurement. A significant difference was found between leptin cord blood and leptin at six months of age $(\mathrm{p}<0.001)$. Significant correlation revealed between delta leptin with WLZ and delta leptin with WAZ at six months of age $(p=0.002$ and $p=0.003$, respectively), and between leptin levels with WLZ $(\mathrm{p}<0.001)$ and leptin levels with WAZ $(p=0.004)$ at six months of age. Leptin levels at the age of six months are lower than umbilical cord blood leptin.

Conclusion: The decreased leptin levels at six months of age are associated with better infant growth.
\end{abstract}

Keywords: leptin; cord blood leptin; infant growth

Permalink/DOI: https://doi.org/10.14710/jbtr.v7i2.11821

\section{INTRODUCTION}

Growth is an increase in body size, mainly in the first thousand days, consisting of intense physiological growth and rapid development of the organs' functions. ${ }^{1}$ Infancy is a critical period where rapid growth may potentially develop future health concerns such as obesity and chronic diseases. Leptin is a hormone that has a direct effect on linear growth. ${ }^{2-4}$ It is produced primarily on white adipose tissue and other tissues, including the placenta, gastrointestinal tract, skeletal muscle, ovaries, breast epithelium, bone marrow, and lymphoid tissues. ${ }^{5-7}$

\footnotetext{
* Corresponding author:

E-mail: dr.mexitalia@gmail.com

(Maria Mexitalia)
}

Arcuate nucleus in the hypothalamus is one of the major targets of leptin. The blood-brain barrier (BBB) at this location is also relatively easy to pass by leptin; thus it plays an essential role in mediating leptin to regulate energy balance, glucose homeostasis, and insulin levels. ${ }^{8,9}$

Leptin was detected in the third trimester of pregnancy, and its levels increased with the development of adipose tissue, then it started to decline at six months old infant. ${ }^{10} \mathrm{~A}$ cohort study in Australia that assessed the association between high umbilical cord leptin level and lower weight gain during infancy was conducted due to a lower increase in adiposity in early infancy. ${ }^{11}$ Meanwhile, a cohort study in the UK that assessed umbilical cord leptin levels and their relation with the 
50 subjects

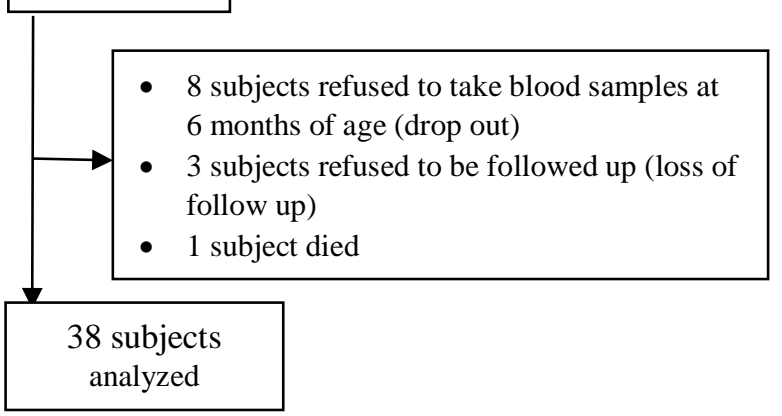

Figure 1. Strobe diagram

weight gain in infancy showed that high umbilical cord leptin levels were associated with slow weight gain at birth and at four months of age. ${ }^{12}$ Another study reported a negative relationship between umbilical cord leptin and infant weight gain up to two years of age, suggesting that leptin has long-term regulating energy balance in early infancy affecting adipose tissues and muscle tissues. $^{9}$ A previous study showed that the overweight/obese children at the age of 2 years had higher leptin levels than those who were not overweight/obese due to leptin resistance. It can lead to weight gain and adipocytes which is larger in late childhood. ${ }^{13}$

However, in Indonesia, the relationship between leptin levels and weight growth, body length, and head circumference of newborn to six-months-old infant has never been studied. Therefore, this study was conducted to determine the relationship between leptin levels and the infant growth age 0-6 months and a part of collaboration research with a topic "Effect of maternal protein intake to intrauterine fetal growth until first six months of life: a study of breastmilk composition, IGF1 levels, and leptin levels".

\section{MATERIALS AND METHODS}

A prospective cohort study was conducted at the Dr. Kariadi Hospital, Diponegoro National Hospital, primary health care centers, and independent-practice-midwives in Semarang between May 2017-August 2018. The subject selection process began during the 3rd trimester (32-<40 weeks) of pregnancy from mothers with single pregnancies and ages $<40$ years, and consecutive sampling was applied. The inclusion criteria were infants born at term, birth weight $>2500$ grams and $<4000$ grams, healthy, age 0-6 months, and exclusively breastfed. The exclusion criteria were infants with asphyxia or respiratory distress, congenital abnormalities, congenital metabolic disorders, and smoking mothers. The drop-out criteria were infants who died or refused to be followed up.

Leptin levels were measured by ELISA methods in the GAKY laboratory, Faculty of Medicine Diponegoro University twice; first, at born, using an umbilical cord blood sample, and second, at six months of age, using a vein blood sample. Infant growth was monitored using WHO 2005 pediatric anthropometric z-scores (Weight for age Z-Score (WAZ), Length for Age -Z score (LAZ), Weight for Length $-Z$ score (WLZ), and Head Circumference for Age -score/HCZ) at born, age of three months, and six months. Delta leptin was defined as the difference between the leptin level at birth and the leptin level at six months of age; meanwhile, delta WAZ, LAZ, WLZ, and HCZ were the differences of measurements between the anthropometric data at birth and the age of six months.

The normality test was conducted to determine the distribution of numeric data. The difference between newborn's anthropometric and cord blood leptin levels with those at six months was analyzed using paired t test

Table 1. The basic characteristics of subjects $(n=38)$

\begin{tabular}{|c|c|c|c|c|}
\hline Variables & $\mathbf{n}$ & $\%$ & Mean \pm SD & $\begin{array}{c}\text { Median } \\
{[\mathrm{min}-\mathrm{max}]}\end{array}$ \\
\hline \multicolumn{5}{|l|}{ Gender } \\
\hline Male & 21 & 55.3 & & \\
\hline Female & 17 & 44.7 & & \\
\hline Maternal gestation age (week) & & & $38.87 \pm 2.21$ & $39[35-43]$ \\
\hline \multicolumn{5}{|l|}{ Newborn anthropometry } \\
\hline Weight (gram) & & & $3120 \pm 349.9$ & $3065[2200-3900]$ \\
\hline Length $(\mathrm{cm})$ & & & $49.2 \pm 1.24$ & $50.1[47.2-53]$ \\
\hline Head circumference $(\mathrm{cm})$ & & & $34.1 \pm 1.56$ & $34.1[31-37.3]$ \\
\hline WAZ & & & $-0.42 \pm 0.86$ & $-0.52[(-2.59)-(1.53)]$ \\
\hline LAZ & & & $-0.16 \pm 0.71$ & $-0.08[(-2.19)-(1.52)]$ \\
\hline WLZ & & & $-0.35 \pm 1.13$ & $-0.28[(-3.49-(2.59)$ \\
\hline \multicolumn{5}{|l|}{ Infant anthropometry at 6 months } \\
\hline Weight (gram) & & & $7183.5 \pm 872.2$ & $7235[5550-8920]$ \\
\hline Length $(\mathrm{cm})$ & & & $65.2 \pm 2.18$ & $65.5[61.3-69.8]$ \\
\hline Head circumference $(\mathrm{cm})$ & & & $42.5 \pm 1.36$ & $42.4[39.8-45]$ \\
\hline WAZ & & & $-0.52 \pm 0.99$ & $-0.48[(-2.31)-(1.67)]$ \\
\hline LAZ & & & $-0.63 \pm 1.09$ & $-0.81[(-2.63)-(1.86)$ \\
\hline WLZ & & & $-0.09 \pm 1.04$ & $0.04[(-2.17)-(2.48)]$ \\
\hline \multicolumn{5}{|l|}{ Leptin } \\
\hline Leptin, cord $(\mathrm{ng} / \mathrm{dL}) \ddagger$ & & & $5884 \pm 1879$ & $6743.4[850-7412.4)$ \\
\hline Leptin, at 6 months $(\mathrm{ng} / \mathrm{dL})$ & & & $2300 \pm 2123$ & $1626.5[414-12345)$ \\
\hline
\end{tabular}

WAZ: weight for age $\mathrm{z}$ score

*Non-normal distributions (non-parametric) 
Table 2. The difference between newborn anthropometric and cord blood leptin with infant anthropometric and leptin level at 6 months

\begin{tabular}{lcccc}
\hline & Newborn & 6 months & p & Delta 6 months \\
\hline WAZ & $-0.42 \pm 0.86$ & $-0.52 \pm 0.99$ & $0.558^{\S}$ & $-0.10 \pm 1.07$ \\
LAZ & $-0.16 \pm 0.71$ & $-0.63 \pm 1.09$ & $0.084^{\S}$ & $-0.46 \pm 1.11$ \\
WLZ & $-0.35 \pm 1.13$ & $-0.09 \pm 1.04$ & $0.278^{\S}$ & $-0.26 \pm 1.40$ \\
Leptin (ng/dL) & $5884 \pm 1879$ & $2300 \pm 2123$ & $<0.001^{\ddagger}$ & $-3584 \pm 2811$ \\
\hline
\end{tabular}

WAZ: weight for age z score

LAZ: length for age $\mathrm{z}$ score

WLZ: weight for length z score

Delta 6 months: difference of anthropometry and leptin between newborn and 6 months of age

$\S$ paired t test $\$$ Wilcoxon test

Table 3. The correlations between cord blood leptin levels and leptin level at 6 months with infant growth at 6 months

\begin{tabular}{lcccc}
\hline & \multicolumn{2}{c}{ Cord Blood Leptin level } & \multicolumn{2}{c}{ Leptin level at 6 months } \\
\cline { 2 - 5 } & $\boldsymbol{r}$ & $\mathbf{p}$ & $\boldsymbol{r}$ & $\mathbf{p}$ \\
\hline WAZ 6 months & -0.086 & 0.607 & 0.457 & $0.004^{\text {s* }^{*}}$ \\
LAZ 6 months & -0.108 & 0.518 & -0.033 & $0.448^{\S}$ \\
WLZ 6 months & -0.031 & 0.854 & 0.584 & $<0.001^{\text {s* }^{*}}$ \\
\hline WAZ: weight for age z score & \multicolumn{2}{c}{ LAZ: length for age z score } & WLZ: weight for length z score \\
§ Spearman's rho correlation. & *Significant (p <0.05) & &
\end{tabular}

Table 4. The correlations between delta Leptin level 0-6 months with delta growth 0-6 months

\begin{tabular}{lcc} 
& \multicolumn{2}{c}{ Delta Leptin Level 0-6 months } \\
\cline { 2 - 3 } & $\boldsymbol{r}$ & $\mathbf{p}$ \\
\hline Delta WAZ 0-6 months & 0.465 & $0.003^{\S^{*}}$ \\
Delta LAZ 0-6 months & 0.124 & $0.457^{\S}$ \\
Delta WLZ 0-6 months & 0.486 & $0.002^{\S^{*}}$ \\
\hline
\end{tabular}

${ }^{\S}$ Spearman's rho correlation. *Significant $(\mathrm{p}<0.05)$

or Wilcoxon test. The correlation between leptin levels and the anthropometric of infants until six months was examined using Spearman's correlation. Statistical significance was defined by $\mathrm{p}$-values $<0.05$. All statistical analysis was performed using SPSS for Windows version 15.

The study was approved by the Ethical Committee of the Faculty of Medicine, Universitas Diponegoro, Semarang, Indonesia (protocol number No. 18/EC/FKUNDIP/2017) and informed consent form was obtained from the parents.

\section{RESULTS}

The study was conducted on 50 subjects who met the inclusion and exclusion criteria. 11 out of 50 subjects refused to be followed up during a study period, and one subject died. A total of 38 subjects have completed the follow-up and were included it in the analysis. The strobe diagram of the research is shown in figure 1 . The characteristics of subjects are shown in table 1 , and it showed that our subjects were normal nutritional status both at the newborn and at the six months old, based on WHO growth standard. ${ }^{14}$

Table 2 showed a significant difference in the mean value of leptin at six months of age $(2300 \pm 2123 \mathrm{ng} / \mathrm{dL})$ than the cord blood leptin levels $(5884 \pm 1879 \mathrm{ng} / \mathrm{dL})$ with $\mathrm{p}<0.001$. Leptin levels decreased significantly at six months of age with the delta leptin $-3584 \pm 2811 \mathrm{ng} / \mathrm{dL}$.

The correlations between the infant's cord blood leptin level and leptin level at six months with growth variables at six months of age were presented in table 3 . There was a significant positive correlation between leptin levels at six months and WLZ ( $p<0.001$ ), and a positive correlation between leptin levels and WAZ at six months. However, there were no correlations between cord blood leptin level with any variables of growth. Table 4 showed the correlation between delta leptin on growth described by delta WAZ, LAZ, and WLZ. This study found that delta leptin was correlated with growth based on the delta WLZ $(r$ 0.486; $\mathrm{p}=0.002)$ and the delta WAZ (r 0.465; $\mathrm{p}=0.003$ ).

\section{DISCUSSION}

Our study showed that the average leptin value for infants-age six months was significantly lower than that at birth. The result was consistent with previous studies that determined leptin levels in children of 0 to 18 months, indicating that serum leptin values decline slowly from birth to 18 months. ${ }^{12,15}$ Previous studies reported that breastfed infants have significantly higher serum leptin levels than formula-fed infants during the first six months of life. It may result from leptin found in mother's breast milk and produced by infant adipocytes, whereas another study stated that leptin is destroyed by the pasteurization process used to produce formula milk. ${ }^{5}$ Other studies have shown a decrease in leptin levels during the first few days of life. The drop in leptin levels may be due to temporary weight loss during this period. A decrease in leptin levels of about $26 \%$ over four months after birth is associated with a $3-6 \%$ weight loss. ${ }^{16-18}$ On the other hand, another study showed that neonatal serum leptin levels did not increase significantly in 21 to 30 days of lactation. ${ }^{19}$ Study in eastern Massachusetts USA found that cord blood leptin was not associated with maternal energy intake but was weakly associated with maternal protein intake. ${ }^{20}$

There was a correlation between delta leptin and delta WLZ and WAZ from birth to six months. It means that leptin correlated with the weight increment and nutritional status. This result is in accordance with several other studies that indicated increasing infant weight was correlated with decreasing leptin levels 
during the first year of life. A low level of cord blood leptin is a solid basis for weight gain in infancy and catch-up growth. ${ }^{9}$ The cohort study in the UK showed that high umbilical cord leptin levels were associated with slow weight gain at four months of age. ${ }^{12}$ Our result showed no correlation between cord blood leptin with the anthropometric status at six months, but the decreasing of leptin level revealed by delta leptin level was correlated with the weight gain. Another study showed a negative correlation between cord blood leptin and fat mass gain at three months of age, especially in infant girls. Likewise, there was a negative correlation between maternal body mass index and fat mass gain in infants measured by air displacement plethysmography. ${ }^{21}$ However, another study that determined the relationship between cord blood leptin levels and BMI from birth to 12 months of age revealed an inverse relationship between cord blood leptin levels and the increase of BMI in the first year of life. ${ }^{22}$ It was similar with the study by Karakosta et al., which found that infants with cord blood leptin $>90$ th percentile were associated with lower weight, height, and BMI at six months of age and thereafter. ${ }^{23}$

Another study showed a positive correlation between delta leptin and delta WLZ, but it did not directly describe the fat mass gain. This result was in accordance with the Iranian study that revealed the positive association between cord blood leptin with birth weight, BMI, and ponderal index of infants. ${ }^{24}$ A previous study also obtained similar results, namely the leptin level positively associated with birth weight. In addition, it was found that the leptin level of appropriate gestational age (AGA) infants were higher than the small for gestational age (SGA) ones, ${ }^{25}$

We also found a significant moderate correlation between leptin levels at six months and WLZ at six months of age. The result was consistent with the study of Wang et al, which reported that the decreased leptin levels in the first year of life positively correlated with better weight gain, ${ }^{26}$ and Chaoimh et al. described that higher cord blood leptin was associated with slower weight gain in early infancy, especially in the first two months of life. ${ }^{11}$ Other studies reported an association of leptin levels with growth, leptin levels at birth associated with WAZ at birth. The changes in WAZ from birth to six months of age has a positive predictive value at six months of age, which is described as a positive relationship between the leptin at six months with the WAZ, LAZ, WLZ, BMI, MUAC, Fat-free mass, fat mass, and fat mass $\%$ age at six months. ${ }^{27}$

Several other hormones such as insulin, IGF-1, growth hormone, adiponectin play a role in metabolic regulation and somatic growth. Similarly, maternal body mass index and breastmilk are related to the regulation of energy expenditure and body weight in infants and later life. ${ }^{28}$ The limitation of this study is that we did not analyze body mass index in mothers, nor the breastfeeding and other biomarkers from cord blood and the six-month-old infants.

\section{CONCLUSION}

In conclusion, leptin levels in infants aged six months are lower than the leptin levels at birth. At six months of age, leptin levels were associated with WAZ and WLZ.
The decreased leptin levels at six months of age are associated to better infant growth. Further studies are needed for a more extended period to evaluate the fat mass and the risk of obesity in childhood related to leptin levels.

\section{ACKNOWLEDGMENT}

This paper is dedicated to memory of the late JC Susanto, MD, Pediatrician.

\section{REFERENCES}

1. Wen X, Gillman MW, Taveras EM, Wen X, Kleinman K, Gillman MW, et al. Childhood body mass index trajectories: modeling, characterizing, pairwise correlations and socio- demographic predictors of trajectory characteristics Childhood body mass index trajectories: modeling, characterizing, pairwise correlations and socio. BMC Med Res Methodol. 2012;12(38):1-13.

2. Murray PG, Clayton PE. Endocrine Control of Growth. Am J Med Genet Part C Semin Med Genet. 2013;163C:76-85.

3. Mook-kanamori DO, Durmus B, Sovio U, Hofman A, Raat H, Steegers EA, et al. Fetal and infant growth and the risk of obesity during early childhood: the Generation R Study. Eur J Endocrinol. 2011;165:623-30.

4. Wang G, Johnson S, Gong Y, Polk S, Divall S, Radovick $S$, et al. Weight Gain in Infancy and Overweight or Obesity in Childhood across the Gestational Spectrum: a Prospective Birth Cohort Study. Sci Rep [Internet]. 2016;6(29867):1-10. Available http://dx.doi.org/10.1038/srep29867

5. Savino F, Rossi L, Benetti S, Petrucci E, Sorrenti M, Silvestro L. Serum Reference Values for Leptin in Healthy Infants. PLoS One. 2014;9(11):1-6.

6. Chiesa C, Osborn JF, Haass C, Natale F, Spinelli M, Scapillati E, et al. Ghrelin, Leptin, IGF-1, IGFBP-3, and Insulin Concentrations at Birth: Is There a Relationship with Fetal Growth and Neonatal Anthropometry? Clin Chem. 2008;54(3):550-8.

7. Mantzoros CS, Magkos F, Brinkoetter M, Sienkiewicz E, Dardeno TA, Kim S, et al. Leptin in human physiology and pathophysiology. Am J Physiol Endocrinol Metab. 2011;301:E567-84.

8. Swieten MMH Van, Pandit R, Adan RAH, Plasse G Van Der. The neuroanatomical function of leptin in the hypothalamus. J Chem Neuroanat [Internet]. 2014;1-14. Available from: http://dx.doi.org/10.1016/j.jchemneu.2014.05.004

9. Much D, Br M, Sedlmeier E, Brunner S, Schmid D, Kristina H, et al. Effect of Reducing the n-6 / n-3 Fatty Acid Ratio on the Maternal and Fetal Leptin Axis in Relation to Infant Body Composition. Obesity. 2014;22:217-24.

10. Savino F, Rossi L, Benetti S, Petrucci E, Sorrenti M, Silvestro L. Serum reference values for leptin in healthy infants. PLOS ONE 2007;9(11): e113024. Available at https://www.ncbi.nlm.nih.gov/pmc/articles/PMC42 40544/ 
11. Chaoimh C, Murray DM, Kenny LC, Irvine AD, Hourihane JOB, Kiely M. Cord blood leptin and gains in body weight and fat mass during infancy. Eur J Endocrinol. 2016;175:403-10.

12. 1Ong KKL, Ahmed ML, Sherriff A, Woods KA, Watts A, Golding J, et al. Cord Blood Leptin is Associated with Size at Birth and Predicts Infancy Weight Gain in Humans. J Clin Endocrinol Metab. 1999;84(3):1145-8.

13. Caroline E, Mantzoros CS, Hughes MD, Rifasshiman SL, Villamor E, Zera CA. Differential associations of leptin with adiposity across early childhood. Obes (Silber Spring). 2013;21(7):14307.

14. World Health Organization. Nutrition Landscape Information System (NLIS) country profile indicators: interpretation guide.WHO Document Production Services Geneva, Switzerland, 2010.

15. Nunes M, Homrich C, Bosa VL, Bernardi JR, Cristina I, Werlang R, et al. Could a remarkable decrease in leptin and insulin levels from colostrum to mature milk contribute to early growth catch-up of SGA infants? BMC Pregnancy Childbirth. 2017;17(410):1-7.

16. Lönnerdal B, Havel PJ. Serum leptin concentrations in infants : effects of diet, sex, and adiposity. Am J Clin Nutr. 2000;72:484-9.

17. Schuster S, Hechler C, Gebauer C, Kiess W, Kratzsch J. Leptin in Maternal Serum and Breast Milk : Association With Infants' Body Weight Gain in a Longitudinal Study Over 6 Months of Lactation. Pediatr Res. 2011;70(6):633-7.

18. Yu X, Rong SS, Sun X, Ding G, Wan W, Zou L, et al. Associations of breast milk adiponectin , leptin , insulin and ghrelin with maternal characteristics and early infant growth : a longitudinal study. Br J Nutr. 2018;120:1380-7.

19. Doneray H, Orbak Z, Yildiz L. The relationship between breast milk leptin and neonatal weight gain. Acta Paediatr. 2009;98:643-7.

20. Mantzoros CS, Sweeney L, Williams CJ, Oken E, Kelesidis T, Rifas-Shiman SL, et al. Maternal diet and cord blood leptin and adiponectin concentrations at birth. Clin Nutr 2010;29:622-6
21. Euclydes VLV, Castro NP, Lima LR, Brito C, Ribeiro L, Simoes FA, et al. Cord blood concentrations of leptin, zinc- $\alpha 2$-glycoprotein, and adiponectin, and adiposity gain during the first $3 \mathrm{mo}$ of life. Nutrition 2018; 54:89-93.

22. Chan D, Goruk S, Becker AB, Subbarao P, Mandhane PJ, Turvey SE, et al. Adiponectin, leptin and insulin in breast milk: associations with maternal characteristics and infant body composition in the first year of life. Int $\mathrm{J}$ Obes [Internet]. 2018;42(1):36-43. Available from: http://dx.doi.org/10.1038/ijo.2017.189.

23. Karakosta P, Roumeliotaki T, Chalkiadaki G, Sarri K, Vassilaki M, Venihaki M. Cord blood leptin levels in relation to child growth trajectories. Metabolism 2016;65(6):874-82.

24. Mellati AA, Mazloomzadeh S, Anjomshoaa A, Alipour M, Karimi F, Mazloomi S, et al.

25. Multiple correlations between cord blood leptin concentration and indices of neonatal growth. Archives of Medical Research 2010;41:26-32.

26. Martinez-Cordero C, Amador-Licona N, GuizarMendoza JM, Hernandez-Mendez J, Ruelas-Orozco G. Body fat at birth and cord blood levels of insulin, adiponectin, leptin, and Insulin-like growth cactor-I in small-for-gestational-age infants. Arch Med Res 2006;37:490-4.

27. Wang L, Mu S, Cheng I, Chen Y, Chen B, Jow G. Decreased leptin concentration in neonates is associated with enhanced postnatal growth during the first year. Kaohsiung J Med Sci [Internet]. 2012;28(10):521-5. Available from: http://dx.doi.org/10.1016/j.kjms.2012.04.013

28. Arsenault JE, Havel PJ, Lo D, Brown KH. Longitudinal measures of circulating leptin and ghrelin concentrations are associated with the growth of young Peruvian children but are not affected by zinc supplementation. Am J Clin Nutr. 2007;86(4):1111-9.

29. Larnkjær A, Ong KK, Carlsen EM, Ejlerskov KT, Mølgaard C, Michaelsen KF.The influence of maternal obesity and breastfeeding on infant appetite- and growth-related hormone concentrations: the SKOT cohort studies. Horm Res Paediatr 2018;90(1):28-38. 